\title{
Representación de la labor científica en dos continentes. DAST comparativo entre niños/as chilenos/as y españoles/as
}

Representation of scientific work in two continents. Comparative DAST between Chilean and Spanish children

Representação do trabalho científico em dois continentes. DAST comparativo entre crianças chilenas / as e espanholas

Teresa VERNAL - VILICIC, Chile

Universidad Finis / tvernal@uft.cl

Lorena B. VALDERRAMA, Chile

Universidad Alberto Hurtado / lvalderrama@uahurtado.cl

Chasqui. Revista Latinoamericana de Comunicación

N. ${ }^{\circ}$ 144, Agosto - Noviembre 2020 (Sección Diálogo de saberes, pp. 259-278)

ISSN 1390-1079 / e-ISSN 1390-924X

Ecuador: CIESPAL

Recibido: 01-04-2020/Aprobado: 04-07-202O 


\title{
Resumen
}

El presente estudio compara la representación social de la labor científica de niños y niñas chilenos y catalanes sobre las personas que desarrollan trabajos científicos. La metodología es un análisis porcentual de los resultados de DAST -Draw a scientist test- que permite conocer desde el dibujo infantil la representación científica que existe de los y las profesionales de las ciencias. A modo de conclusión fue posible establecer que no existió una gran diferencia sobre la imagen estereotipada de los profesionales de la ciencia. Sin embargo tanto las niñas chilenas como españolas dibujaron más mujeres que los niños participantes, dejando abierta la mirada de género frente a esta técnica para estudios posteriores. .

Palabras clave: comunicación científica, educación científica, estereotipos, percepción estudiantil

\begin{abstract}
The study compares the social representation of the scientific work by Chilean and Catalan childrens. The methodology is a percental analysys of the DAST -Draw a scientist test- that allows to know from the drawing the childhood representation that exists of the professionals of the sciences. In conclusion was possible to establish that there was not a large difference on the stereotyped image of the professionals of science. However, one of the most significant results was that both Chilean and Spanish girls draw more women scientist than the boys, leaving the gender perspective open to this technique for further studies.
\end{abstract}

Keywords: comunicação científica, educação científica, estereotipos, percepção do aluno

\section{Resumo}

O presente estudo compara a representação social do trabalho científico de crianças chilenas e catalãs sobre as pessoas que desenvolvem trabalhos científicos. A metodologia é uma análise percentual dos resultados do DAST Desenha um teste cientifico - que permite conhecer desde o desenho infantil a representação científica que existe dos profissionais das ciências. Como conclusão, foi possível estabelecer que não havia grande diferença na imagem estereotipada dos profissionais da ciência. No entanto, tanto as meninas chilenas quanto as espanholas atraíram mais mulheres do que as crianças participantes, deixando a perspectiva de gênero aberta a essa técnica para mais estudos.

Palavras-chave: scientific communication, science education, stereotypes, student perception 


\section{Introducción}

Los medios de comunicación desempeñan un rol preponderante en la configuración de las percepciones y representaciones sociales sobre el quehacer científico, creando y reforzando estereotipos en la población infantil (Schibeci, 1986; Ruiz-Mallén y Escalas, 2012; Valderrama et al., 2016). Desde la década de 1950 es posible encontrar investigaciones sobre percepción y representación de la labor científica. Dichos estudios advertían de la existencia de una imagen estereotípica en la población juvenil en relación a las personas dedicadas a las ciencias: hombre de mediana o avanzada edad, con bata blanca y lentes, que trabajaba en un laboratorio realizando experimentos químicos (Mead \& Metraux, 1957; Beardslee y O'Dowd, 1961). Desde entonces, se han estudiado los estereotipos infanto-juveniles en distintos países, como Estados Unidos, Australia, Inglaterra, Canadá, España, Portugal, Francia, Italia, República Checa, Polonia, Rumania, Grecia, Turquía, Singapur, China, India y Corea del Sur (Chambers, 1983; Schibeci \& Sorensen, 1983; Schibeci \& Riley, 1986; Fort \& Varney, 1989; Flick, 1990; Mason et al., 1991; Finson et al., 1995; Sumrall, 1995; Neathery, 1997;Newton \& Newton, 1998; She, 1998; Song \& Kim, 1999; Andre et al., 1999; Barman, 1999; Joyce \& Farenga, 1999; Weinburg \& Steele, 2000; Calabrese Barton, 2001; Morgan, Isaac \& Sansone, 2001; Chavous, 2002; Finson, 2001, 2002; Fung, 2002; Mattern \& Shau, 2002; Rubin, Bar \& Cohen, 2003; Weinburg, 2003; Buldu, 2006; Christidou, 2006; Rodari, 2007; Chavous et al., 2008; Christidou, 2011; Christidou et al., 2012; Ruiz-Mallén \& Escalas, 2012; Erten et al., 2013; Narayan, 2013; Toğrol, 2013; Chen, \& Cowie, 2013; Zhai et al., 2014; Woods-Townsend et al., 2015). En Latinoamérica también se han realizado estudios de este tipo en Bolivia, México, Uruguay, Colombia, Argentina y Chile (González et al., 2009; Polino, 2011; Pujalte et al., 2014; Bravo et al., 2014, Vernal, 2014; Valderrama et al., 2016) Estudios comparados han sido más escasos, concentrándose principalmente en países de habla inglesa, pese a que su riqueza radica entregar información sobre la representación social de la ciencia situada en sus contextos socioculturales (Farland-Smith, 2017). En la década de los años ochenta se realizó un estudio comparado de la población infantil de Canadá, Estados Unidos y Australia para analizar la imagen estereotípica de las ciencias y las personas que se dedican al quehacer científico (Chambers, 1983). Los resultados dieron cuenta que la imagen estereotípica constatada en estudios previos, se mantenía en los niños y las niñas de entre 5 y 11 años de habla inglesa pertenecientes a distintos continentes.

En cuanto a las técnicas utilizadas, estas han variado entre cuestionarios, ensayos, entrevistas y dibujos, principalmente. En la segunda mitad de la década de 1970 se diseñó el Draw A Scientist Test, conocido como DAST para estudiar las representaciones sociales de las ciencias en la población infantil y determinar estereotipos presentes en niños y niñas en cuanto al género, edad, atributos personales e indumentaria de las personas dedicadas a las ciencias. Esta 
herramienta consiste en solicitarles a niños y niñas que dibujen a una persona que haga ciencias, permitiendo con ello identificar estereotipos infantiles sobre el quehacer científico, presentando a su vez desafíos metodológicos (Reinisch et al., 2017), por lo que Toma et.al (2018) elaboraron una propuesta de mejoramiento a la metodología DAST y a su protocolo.

A través de los años y de su aplicación en diferentes contextos socioculturales, el DAST se ha modificado analizando, cada vez más, categorías y estereotipos como la raza/etnia, el género y los factores de riesgo (Schibeci \& Sorensen, 1983; Fort \& Varney, 1989; Flick, 1990; Finson et al., 1995; Sumrall, 1995; Finson, 2001, 2002; Rodari, 2007; Ruiz-Mallén \& Escalas, 2012; Meyer et.al, 2019).

\section{Metodología}

\subsection{DAST comparativo}

El presente estudio buscó, a partir de un enfoque cuantitativo de la técnica DAST (Toma, et. al, 2018), establecer una comparación a nivel cultural de la representación social que tiene la población adolescente sobre la labor científica. Se estudió a dos grupos de escolares en Chile y España que, a pesar de encontrarse en continentes distintos, provenían de un entorno social similar. Ambos grupos son de escolaridad pública, pertenecientes a paises democráticos e interconectados entre sí. Esto último, puesto que Chile y España, mantienen relaciones bilaterales desde hace más de 30 años y, además, intercambios a nivel investigativo y científico (MINREL, 2020).

Tomando en cuenta dichos antecedentes se decidió definir y comparar la percepción que tienen los niños y las niñas adolescentes en Chile y Cataluña en España de las personas que hacen ciencias, es decir caracterizar la imagen estereotipada de las mismas, a partir del género y la nacionalidad de los/as participantes. Todo ello para reconocer qué diferencias y similitudes podrían existir de acuerdo a los actores y a las prácticas en la labor científica entre grupos de adolescentes que se movilizan en realidades paralelas pero en continentes distintos.

En relación a establecer una base metodológica, es posible decir que no han sido muchos los estudios de estilo comparativo entre culturas y etnias, especialmente a nivel latinoamericano y europeo (Medina, Middleton \& Orihuela, 2011) que utilicen el dibujo como una metodología gráfica y proyectiva relevante para la representación infantil de las ciencias (Valderrama et al., 2016)

Finson (2003) realizó un análisis comparativo de DAST en diferentes grupos raciales y posteriormente Medina, Middleton y Orihuela (2011) compararon la visualización de la imagen las personas que hacen ciencias entre niños/as de países latinoamericanos como Colombia y Bolivia, siendo ésta una de las pocas referencias de DAST comparativo entre hispanohablantes. Otros antecedentes 
de DAST comparativo utilizan muestras de públicos jóvenes pero pertenecientes a los mismos sectores geográficos (Finson, Pedersen \& Thomas, 2006).

Desde una perspectiva gestáltica (Oaklander, 2008) el dibujo es una de las primeras destrezas del desarrollo infantil, por tanto, permite generar mayor confianza entre los/as participantes y los/as investigadores/as. Así como, también, logra identificar las imágenes que tiene la población infantil con respecto a las ciencias, aunque no permiten indagar en la causa de dicha imagen (Manzoli, Castelfranchi, Gouthier \& Cannata, 2006).

\subsection{Muestra}

Con el consentimiento informado, la previa autorización de los tutores correspondientes y el asentimiento de los participantes, tal como indica la Tabla 1, se recolectaron y analizaron - en 2016 - un total de 191 dibujos de niños/ as y adolescentes chilenos y españoles con edades fluctuantes entre los 12 y los 16 años pertenecientes a dos escuelas de Antofagasta en Chile y Barcelona en España.

Ambos establecimientos educacionales - Liceo Bicentenario Andrés Sabella de Antofagasta en Chile e Institut Public Rovira Forns de Barcelona en España - fueron escogidos previamente para el estudio, debido a sus similitudes geográficas, sociales y generacionales (Hernández, Fernández y Baptista, 2010). Los grupos analizados corresponden a niños/as integrantes de familias pertenecientes a grupos socioeconómicos medios que participan de un entorno sin riesgo social e integran cursos mixtos (hombres y mujeres) de alta exigencia académica.

Tabla $\mathbf{N}^{\circ} \mathbf{1}$ : Distribución de la muestra de los 191 dibujos por país, género y edades.

\begin{tabular}{|c|c|c|c|c|c|}
\hline & \multicolumn{2}{|c|}{ Chile (N=94) } & \multicolumn{2}{c|}{ España (N=97) } & \\
\hline Edad & Hombre & Mujer & Hombre & Mujer & Total \\
\hline 12 & 0 & 0 & 0 & 15 & 15 \\
\hline 13 & 10 & 19 & 26 & 18 & 73 \\
\hline 14 & 11 & 11 & 7 & 7 & 36 \\
\hline 15 & 11 & 11 & 7 & 11 & 36 \\
\hline 16 & 10 & 11 & 5 & 1 & 27 \\
\hline Total & 42 & 52 & 45 & 52 & 191 \\
\hline
\end{tabular}

\subsection{Categorización: actores y prácticas}

Tanto en Chile como en España se solicitó a los/as participantes que dentro del aula, en horario libre de clases y bajo la supervisión de un/a profesor/a, dibujaran a "una persona que hace ciencias" y no "a un científico" (Valderrama et al., 2016) con la finalidad de no direccionar la imagen en relación a género, nacionalidad $u$ área de trabajo. Una vez recolectados aquellos dibujos que 
cumplieron con el asentimiento y consentimiento de los tutores, éstos fueron separados por geografía y género, construyendo en paralelo las categorías de análisis que se dividieron entre actores y prácticas, tal como se explica en la Tabla 2. No se realizó un análisis por rango etario, ya que existieron ciertas diferencias numéricas de dibujos por edades. No obstante, se consideró como un todo al grupo adolescente de acuerdo al género y al país de origen.

Las subcategorías básicas de los actores y prácticas analizadas en los dibujos, orientadas a la típica imagen del científico - hombre de mediana edad, vello facial, bata blanca y anteojos que se dedica a la química o biología en un laboratorio con instrumentos como probetas o tubos de ensayo - fueron seleccionadas de acuerdo a estudios iniciales de DAST que se han mantenido en análisis posteriores (Mead y Metraux, 1957; Beardslee \& O’Dowd, 1961; Chambers, 1983; Flick, 1990; Finson et al. 1995; Ruiz-Mallén \& Escalas 2012). A ello se sumaron subcategorías emergentes que nacieron de los dibujos analizados y que, previamente, no fueron objeto de análisis como por ejemplo personajes no humanos, apariencia extravagante, actitud seria o desarrollo de trabajo colectivo. Los dibujos, entonces, fueron codificados por el equipo de la investigación, quienes tenían experiencia previa en el análisis de datos de la herramienta DAST y DAST-C, determinando, así, con un número 1 o un o las categorías mencionadas que estaban presentes o ausentes en cada una de las 191 imágenes (Ruiz-Mallén \& Escalas 2012).

Posteriormente se realizó una cuantificación en porcentaje para cada categorización, según país, género y total de dibujos. Luego se procedió a realizar una comparación de acuerdo a los porcentajes obtenidos en cada código, en relación al género y nacionalidad de los participantes. Todo ello permitió graficar los resultados y finalmente, establecer una conclusión descriptiva.

Tabla $\mathbf{N}^{\circ}$ 2: Categorías de metagrupos distribuidas entre actores y prácticas.

\begin{tabular}{|c|c|c|c|}
\hline & Categorías & $\begin{array}{l}\text { Subcategorías } \\
\text { predeterminadas }\end{array}$ & Subcategorías emergentes \\
\hline \multirow{6}{*}{ Actores } & Sexo & Mujer/ Hombre & Sexo indefinido/ No humano \\
\hline & Edad & Joven/Mayor & \\
\hline & Nacionalidad & & $\begin{array}{l}\text { Chilena/Española/ } \\
\text { Extranjero }\end{array}$ \\
\hline & Apariencia & $\begin{array}{l}\text { Bata blanca/Vello facial/ pelo enmarañado/ } \\
\text { gafas }\end{array}$ & Extravagante/ Exitoso \\
\hline & $\begin{array}{l}\text { Personalidad y } \\
\text { actitud }\end{array}$ & $\begin{array}{l}\text { Amigable/no amigable/solitario/loco/violento/ } \\
\text { atormentado/sonriente/maléfico }\end{array}$ & $\begin{array}{l}\text { Con otras personas/con } \\
\text { personajes imaginarios/serio }\end{array}$ \\
\hline & Áreas & $\begin{array}{l}\text { Bioquímica/astrofísica/Matemáticas/ } \\
\text { Medicina/Medio Ambiente/Tecnología/Artesy } \\
\text { Humanidades/áreas indefinidas }\end{array}$ & \\
\hline Prácticas & Herramientas & $\begin{array}{l}\text { Fórmulas/ Herramientas de laboratorio/ } \\
\text { Herramientas tecnológicas/Sin presencia }\end{array}$ & Lápiz en el bolsillo \\
\hline
\end{tabular}




\begin{tabular}{|l|l|l|l|}
\hline & Locación & $\begin{array}{l}\text { Laboratorio/observatorio/oficinas/ } \\
\text { sala de clases/aire libre/ indefinido }\end{array}$ & \\
\hline & Tipo de actividad & $\begin{array}{l}\text { Experimentando/ pensando/ enseñando/ } \\
\text { indefinido }\end{array}$ & \\
\hline & Factores de riesgo & Peligro/ orden/seguridad & \\
\hline
\end{tabular}

\subsubsection{Criterios de codificación: DAST}

Las características personales del personaje científico dibujado se reconocieron desde el sexo, edad y nacionalidad, en subcategorías predeterminadas como SCMAN para hombres, SCWOMAN para mujeres (Mead y Metraux, 1957), SCAGE para las personas que hacen ciencias aparentemente de edad joven y SELDER de edad media en adelante. La subcategoría NO HUMAN se utilizó para aquellos personajes que no eran de especie humana y N/A para sexo indefinido. Asimismo, en esta oportunidad, se definió como CHILEAN, SPANISH or FORENGEIR la nacionalidad de las personas dibujadas, a partir de la presencia de banderas o frases escritas.

Para evidenciar la apariencia física de los personajes de cada dibujo, también, se codificó de acuerdo a la presencia de WHITECOAT, GLASSES, BEARD/ MUSTACHE and DIVESHED HAIR (Chambers, 1983). Como subcategoría emergente se notó, en algunas imágenes, la presencia de extravagancias que fueron diferenciados como FASHION/EXTRAVAGANT.

En paralelo se codificó actitud y personalidad de las personas que hacen ciencias como UNDFRINDLY, FRIENDLY or SOLITARY. En caso de estar acompañados por otra persona se utilizaron categorías emergentes como WITH OTHER PEOPLES and WITH FICTIONAL CHARACTERS. También se analizaron las actitudes y el tipo de trabajo que se encontraban realizando.

Las áreas de investigación, herramientas de trabajo y locaciones se definieron en la codificación de los datos de acuerdo al entorno y actividad desempeñada de acuerdo a la propuesta de las autoras Ruiz-Mallén \& Escalas (2012). En este caso se codificó de acuerdo a las profesiones como BIOCHEM para bioquímica, ASTROPHYS para astrofísica, MATH para matemáticas, ENVIRON para medio ambiente, MEDICINE para áreas de la salud, TECHNOL para áreas tecnológicas, ART\&HUM para artes y humanidades, además de N/A para áreas indefinidas dentro del dibujo.

En cuanto a las herramientas de trabajo estas se analizaron con los códigos FORMULA/PHRASES para la aparición de números, códigos o palabras, LABTOOL para la presencia de instrumentos como matraces, probetas o tubos de ensayos, entre otros y TECHTOOL para ordenadores, tablets $u$ otros similares. N/A correspondió a la ausencia de herramientas de trabajo. También se definieron subcategorías para locaciones, a partir del espacio escogido por los/as dibujantes donde se encontraban los profesionales de las ciencias desarrollando sus labores. 
Los valores humanos y sociales se definieron a partir de una actitud representado en códigos tales como CRAZY, VIOLENT, TORMENTED, SMILING, MALEFIC or SERIUS, éste últimos creado por las investigadoras, ya que la expresión rictus fue recurrente en los dibujos presentados. Asimismo se consideraron los factores de riesgo diferenciados en DANGER para entornos complejos de los personajes dibujados o donde existiera manipulación de artefactos o sustancias peligrosas. También se consideró CLEAN/TIDY or SAFETY para entornos seguros y fuera de complicaciones.

\section{Resultados}

\subsection{Apariencia personal}

Un 77\% del total de los dibujos de ambas nacionalidades contó con la presencia de científicos de sexo masculino, mientras que sólo un $23 \%$ de las imágenes representó a mujeres científicas. Dicha tendencia por el sexo femenino estuvo representada mayormente por participantes mujeres, tanto chilenas (37\%) como españolas (40\%). Asimismo el $2 \%$ de los/as participantes, chilenos y españoles, dibujó personajes no humanos y ficticios.

La tendencia a dibujar jóvenes fue de un $64 \%$ del total de los dibujos, donde los/as participantes chilenos predominaron esta opción en un $77 \%$ del total de sus dibujos. En cuanto a la nacionalidad de los personajes dibujados, el $21 \%$ de los dibujos españoles mostraron presencia de extranjeros y en Chile, paralelamente, esta presencia se visualizó en sólo el $3 \%$ de los dibujos. No obstante, el $5 \%$ de los dibujos chilenos representó a las personas locales que hacen ciencias y sólo un $2 \%$ de los dibujos españoles contaron con la presencia de profesionales del mismo país. Dicha opción se manifestó de manera voluntaria en los participantes, ya que no se solicitó definir nacionalidad en los dibujos.

La presencia de una imagen estereotipada, en el total de los dibujos, tanto bata blanca $(73 \%)$, pelo enmarañado $(47 \%)$, gafas $(48 \%)$ y bigotes $(35 \%)$ tuvo una presencia similar en ambas naciones, siendo las niñas chilenas quienes tuvieron menos tendencia a dibujar personas con bigotes, lo que se representó en un 13\% del total de la muestra del país (Ver Gráfico $\mathrm{N}^{\circ} 1$ ). Curiosamente un $20 \%$ del total de los dibujos, presentó un lápiz en el bolsillo superior del delantal, lo que se consideró como un código emergente. Existió, además, la representación de personajes extravagantes que utilizaban atuendos coloridos, tacones, gorras entre otros. Todos ellos fueron personificados en un $22 \%$ de dibujos chilenos y en un $31 \%$ de dibujos españoles. 
Gráfico $\mathbf{N}^{\circ} 1$ : Apariencia de la persona que hace ciencias.

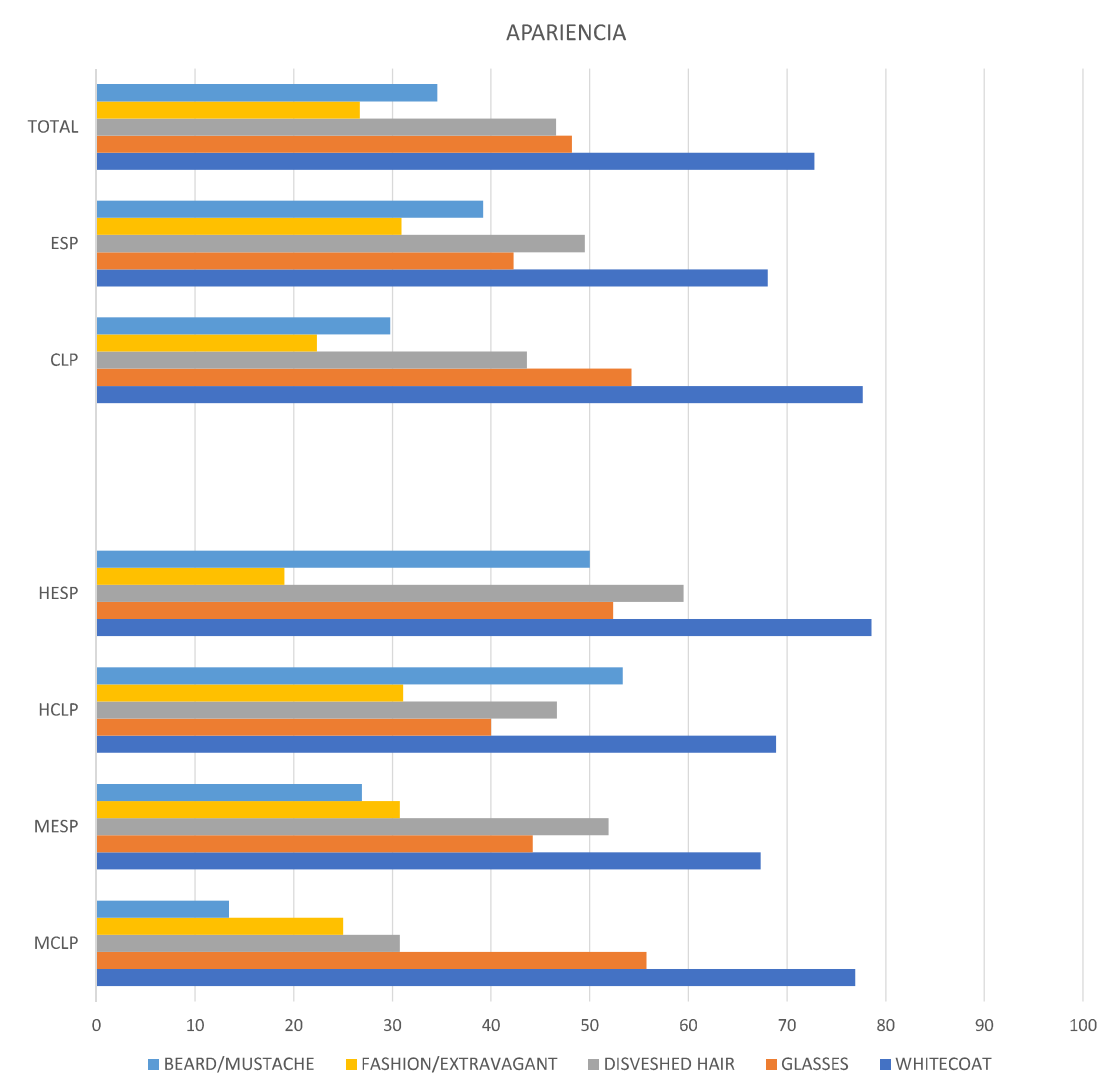

\subsection{Herramientas de conocimiento científico}

En cuanto a las áreas de desarrollo, la bioquímica fue la más escogida, tanto en dibujos chilenos (60\%) como en dibujos españoles (60\%), logrando un $60 \%$ del total de todos los dibujos y no evidenciando diferencias entre preferencias por país, ni género. El área de medio ambiente logró sólo el 1\% del total de los dibujos, mientras que la astrofísica fue representada por el $2 \%$ de los dibujos, solamente, provenientes de Chile. Las matemáticas estuvieron presentes con un $7 \%$ del total de los dibujos, la medicina con un $5 \%$ y la tecnología con un $2 \%$, sin mayor variación entre ambos países.

Un $38 \%$ de la muestra chilena no dibujó un área de trabajo científico y sólo un $5 \%$ de los dibujos españoles dejó indefinida el área de trabajo. Ningún dibujo representó las artes y humanidades como una labor científica y, en tanto, las multidisciplinas estuvieron representadas por el 1\% del total de los dibujos. 
Gráfico $\mathbf{N}^{\circ}$ 2: Herramientas de Investigación de la persona que hace ciencias.

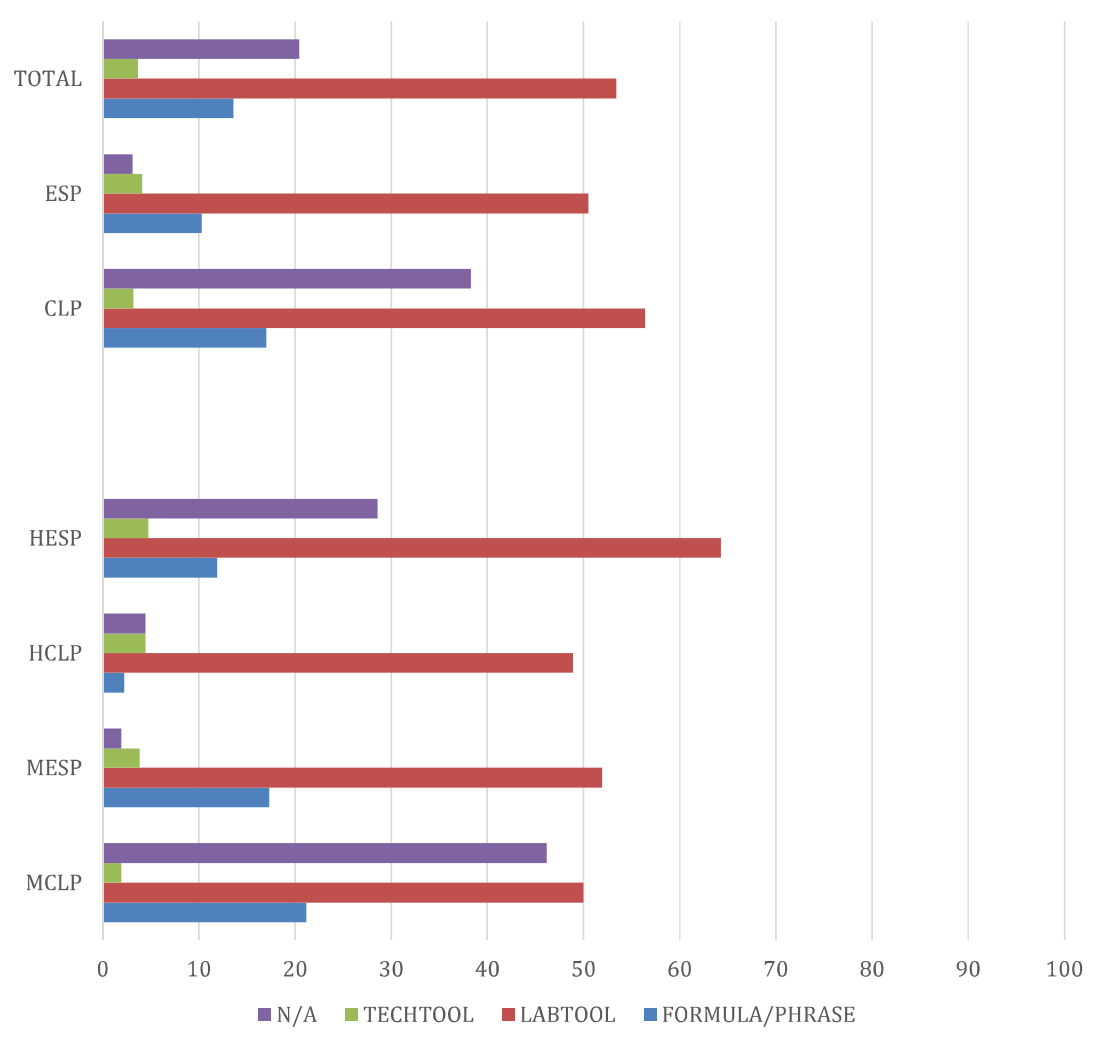

En el Gráfico $\mathrm{N}^{\circ} 2$ se pueden observar las herramientas de trabajo, siendo las más escogidas y sin variación las herramientas de laboratorio (53\%), tales como matraces, tubos de ensayo y probetas. Mientras que sólo el $4 \%$ del total de los dibujos se refirió a herramientas tecnológicas como ordenadores, Ipad o telefonía móvil.

En cuanto a las locaciones, el laboratorio fue la más escogida por los/as participantes de ambas nacionalidades, pues el $28 \%$ de las imágenes chilenas y el $41 \%$ de las imágenes españolas representaron la labor científica en dicho espacio. Sin embargo, no hubo presencia de la categoría biblioteca para definir el entorno de trabajo. Igualmente y sin variaciones, estuvieron presentes las categorías de aire libre y de oficina, sólo con el 1\% propiamente tal. En paralelo un $5 \%$ de la muestra española no definió una locación de desempeño para la labor científica, a diferencia de la muestra chilena donde sólo un $9 \%$ demostró locación indefinida. 


\subsection{Valores humanos y sociales de las personas que hacen ciencias}

En el Gráfico №3 se puede observar que un 20\% del total de los dibujos representó personas poco amigables que se dedican a hacer ciencias, mientras que un $59 \%$ definió a estas personas como amigables y cercanas. Lo anterior no demostró grandes diferencias entre países comparados.

En cuanto al trabajo en equipo, tanto en Chile como en España, hubo presencia de un $1 \%$ de trabajo en equipo, es decir de más de una persona dedicada a la actividad científica en un mismo espacio y un 1\% acompañó al personaje principal de un personaje de ficción.

Gráfico No3: Personalidad de la persona que hace ciencias

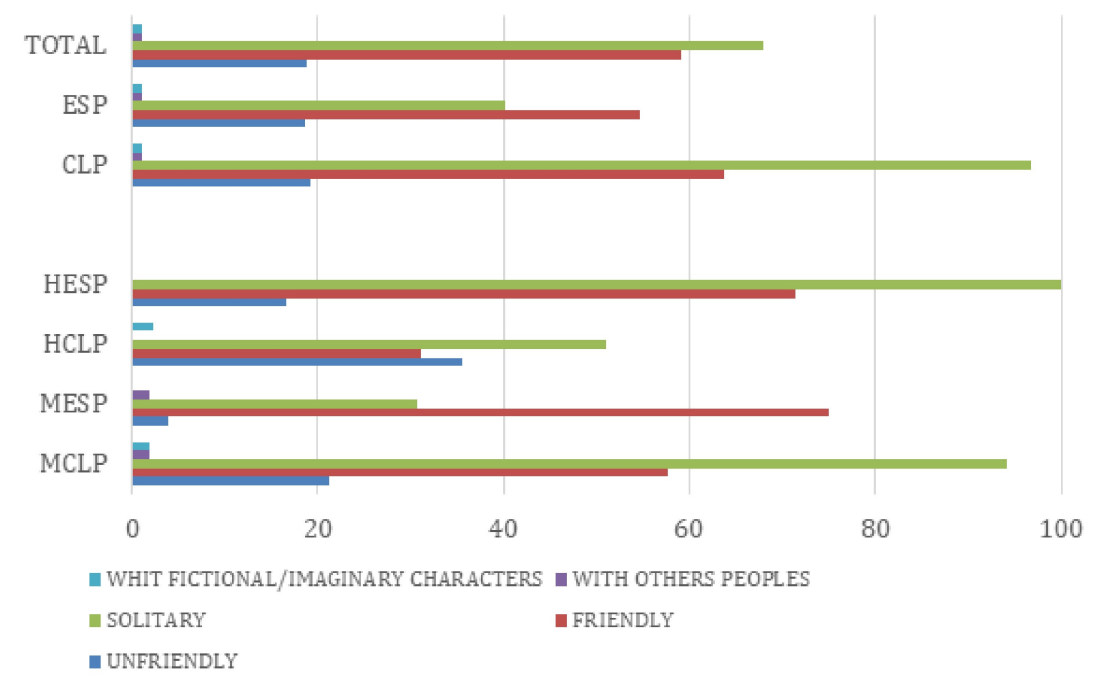

Un 47\% del total de los dibujos representó a las personas que hacen ciencias realizando acciones indefinidas, mientras que el $19 \%$ de la muestra chilena y el $4 \%$ de la muestra española estuvieron conformadas por personas en acción pensativa. Sólo el $2 \%$ de niños chilenos y el $2 \%$ de niños españoles dibujaron a personas enseñando, a diferencia de las niñas que superaron el $10 \%$ en sus respectivas muestras. En tanto, un $45 \%$ de los/as participantes españoles definieron la opción de experimentar, mientras que los/as chilenos/as sólo obtuvieron un $29 \%$ de selección para dicha categoría.

\subsection{Factores de riesgo}

En cuanto a las actitudes, el $63 \%$ de los personajes dibujados, sin diferencias entre países, representaron rostros sonrientes. No obstante un $30 \%$ dibujó 
rostros serios, un $9 \%$ peligrosos, un $15 \%$ con gestos "alocados" y un $5 \%$ maléficos. No hubo presencia de actitud violenta en los dibujos chilenos, a diferencia de España que presentó la violencia en un $3 \%$, predominantemente en los dibujos de los niños. La imagen atormentada se evidenció en un $11 \%$ de los dibujos españoles y en un $1 \%$ de los dibujos chilenos.

En cuanto a los factores de riesgo observados en el Gráfico No 4 , estos sólo estuvieron presentes en un $9 \%$ del total, representado en dibujos con ambientes peligrosos, donde había presencia de explosiones o gases tóxicos. Un 39\% de los dibujos españoles mostraron ambientes limpios y ordenados, mientras que sólo un $4 \%$ de los dibujos chilenos coincidieron con esta categoría. Asimismo un $28 \%$ de dibujos españoles presentaron escenarios seguros y un $1 \%$ de los chilenos definieron esta opción. Finalmente un $80 \%$ de dibujos chilenos fueron imprecisos en cuanto a los factores de riesgo, mientras que ningún dibujo español demostró imprecisión en esta línea.

Gráfico $\mathbf{N}^{\circ} 4$ : Factores de riesgo del trabajo realizado por la persona que hace ciencia

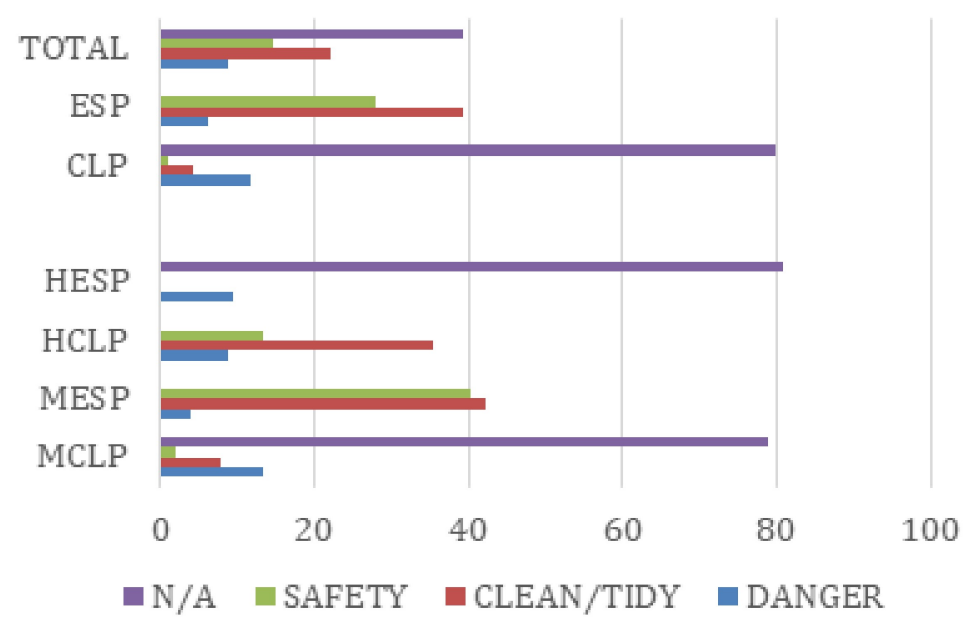

\section{Conclusiones}

De acuerdo al análisis comparativo realizado fue posible determinar que las diferencias entre ambas muestras no fueron tan notorias, aunque se percibieron algunas variantes entre nacionalidades. Fue posible concluir que tanto en los dibujos realizados en Chile como en España, existió una tendencia a dibujar científicos hombres, algo que ha ocurrido en estudios previos realizados en las localidades estudiadas (Valderrama et al., 2016; Ruiz-Mallén \& Escalas 2012) y en estudios de otras regiones (Finson, 2002; Meyer et.al, 2019). 
El panorama desigual sobre la presencia de mujeres en el campo cientifico es similar en ambos paises, pues en España sólo un 20\% de investigadoras ocuparía cargos directivos en centros y un 35\%, estaría a cargo de proyectos (CSIC, 2020). En Chile, en tanto, los centros de investigación son dirigidos en un 16\% por mujeres y un 27\% de proyectos, sería liderado por mujeres (CONICYT, 2017).

Los medios de comunicación desempeñan un rol crucial cuando presentan a personas que se dedican al quehacer científico, influyendo en la imagen de la mujer en las ciencias (Steinke et al., 2008). La prensa y los medios de comunicación estarían apoyando poco en revertir esta imagen, dado que, al menos en la prensa británica, las mujeres son menos citadas que los hombres (Kitzinger et al, 2008a, 2008b) y, al menos en la prensa española, las mujeres protagonizan sólo el 14,3\% de las noticias científicas, frente al 70,6\% de los artículos, protagonizados por científicos hombres (Aladro, et. al, 2014). No obstante, tanto las niñas chilenas como españolas dibujaron más mujeres que los niños participantes, aunque no menos hombres, algo que marca un leve cambio con los primeros estudios de este tipo donde sólo las niñas dibujaban mujeres científicas (Chambers, 1983).

Asimismo para los y las adolescentes chilenos/as la imagen de personas jóvenes o de mediana edad que hagan ciencias es más predominante que en España, esto coincide con estudios previos realizados en el mismo país (González et al., 2009; Valderrama et al., 2016). La mirada de mundo también varía, ya que siendo España un país con mayor conexión a nivel geográfico hubo tendencia a dibujar más extranjeros que en Chile. En tanto la apariencia física de las personas que hacen ciencias representadas en el marco de otros estudios similares realizados en los últimos años en ambos países (Ruiz-Mallén \& Escalas, 2012; Valderrama et al., 2016), tales como bata blanca, pelo enmarañado, gafas y bigotes se manifestaron en igual medida en ambas locaciones. Algunos investigadores han señalado que esta imagen estereotípica de las personas que hacen ciencia puede guardar relación con los personajes ficcionales de la industria cultural (Schibeci \& Sorensen, 1983; Weingart y Pansegrau, 2003; Steinke et al., 2008, Rodari, 2007). En cuanto a la labor desarrollada por las personas que hacen ciencias se presentó una coincidente preferencia por la bioquímica en ambas nacionalidades, sin distinción. Todo ello ha ocurrido en estudios previos realizados en ambas nacionalidades (Valderrama et al., 2016; Ruiz-Mallén \& Escalas, 2012)y, también, en otros estudios de DAST comparativos, como el de Medina, Middleton y Orihuela (2011). Mientras que hubo una baja predilección para las matemáticas, medicina, medio ambiente y tecnología. Sólo en los dibujos chilenos hubo presencia de la astrofísica, seguramente debido al contexto de la muestra compuesta por una amplia presencia de observatorios astronómicos en la zona (Vernal, 2015). Pese a ello fue una presencia bastante baja.

En ambos países no se presentaron a las ciencias sociales como parte del quehacer científico. Asimismo para todo el grupo estudiado las herramientas 
más utilizadas en las ciencias serían las de laboratorio bio-químico, mientras que las herramientas tecnológicas presentaron baja presencia. Esto presenta una diferencia con estudios realizados a estudiantes de edades inferiores (6 a 10 años) en estudios europeos (Rubbia et al., 2015). En paralelo, el laboratorio bio-químico como locación para desarrollar ciencias fue el más dibujado por ambos grupos y los lugares menos asociados a las ciencias fueron la biblioteca, el aire libre y la oficina, sin distinción. Ambos aspectos se condicen con estudios previos realizados en Cataluña (Ruiz-Mallén \& Escalas, 2012). En relación a la personalidad dibujada, en general, hubo una mayor asociación a la amistad y cercanía. Tampoco se avizoraron diferencias en relación al trabajo en equipo, ya que se asoció el quehacer científico al trabajo en solitario, como en estudios previos realizados en ambos países (González et al., 2009; Ruiz-Mallén \& Escalas, 2012; Valderrama et al., 2016). No obstante, sí se visualizaron diferencias en cuanto a la acción de enseñar, ya que tanto niñas chilenas como españolas tuvieron una mayor tendencia a dibujar personas enseñando, a diferencia de ambos grupos de niños.

En relación a los factores de riesgo, en ambos grupos fueron superiores a los factores de riesgo dibujados en estudios previos (Chambers, 1983), pero se presentaron más escenarios seguros del quehacer científico y personas que hacen ciencias sonrientes. La violencia, sin embargo, sólo se manifestó en un pequeño porcentaje de dibujos españoles.

Un punto que llamó la atención y marcó una diferencia realmente notoria fue que en los dibujos españoles hubo mayor presencia de espacios limpios, ordenados y seguros, lo que puede referirse al nivel de desarrollo de España frente a Chile.

Finalmente, si bien, se esperaba encontrar diferencias más notorias relacionadas con la variedad de áreas científicas, la imagen estereotipada de la persona que hace ciencias que tienen los niños y las niñas de Chile y España participantes en este estudio es realmente similar. Este escenario, posiblemente, tenga relación con las realidades culturales de ambos paises que han vivenciado dictaduras en su historia política y donde, además, la religión católica es predominante, siendo la visión conservadora, probablemente, un factor influyente en la percepción de la mujer en la ciencia.

Por ello, es importante generar políticas públicas que permitan revertir estos estereotipos porque limitan las representaciones de las áreas de investigación y prácticas que conforman el quehacer científico, (Chernobilsky, 2006), como también de las personas que se dedican a esta labor. Como han demostrado estudios previos, la representación social de las ciencias explica los estereotipos de género que existen en esta área profesional (Miller, Eagly \& Linn, 2014). Dentro de las limitaciones, tal como se menciona al comienzo del documento, los estudios DAST comparativos no son suficientes como para establecer relaciones entre estudios previos. Para investigaciones futuras en la misma línea sería interesante desarrollar comparaciones entre realidades 
escolares diferentes, tanto a nivel geográfico como social. Un ejemplo de ello sería el estudio de Buldu (2006), donde si bien no fue un estudio comparativo se consideraron las diferencias de percepción desde los entornos socioeconómicos de niños y niñas en Turquía lo que permitiría, probablemente, obtener mayores matices entre grupos participantes.

\section{Apoyos}

Esta investigación contó con la colaboración, sin fines de lucro, del Observatorio de Difusión de la Ciencia de la Universidad Autónoma de Barcelona, UAB.

\section{Referencias}

Aladro, E., Padilla, G., Requeijo, P., Semova, D., García, J., García M.T. \& M. Viñarás (2014), La presencia y representación de la mujer científica en la prensa española, Revista Latina de Comunicación Social, 69, 176-194.

Andre, T., Whigham, M., Hendrickson, A. \& Chambers, S. (1999). Competency beliefs, positive affect and gender stereotypes of elementary students and their parents about science versus other school subjects. Journal of Research in Science Teaching, 36, 719-747. DOI: 10.1002/(SICI)1098-2736(199908)36:6<719::AID-TEA8>3.0.CO;2-R

Barman, C. R. (1999). Students' views about scientists and school science: Engaging K-8 teachers in a national study. Journal of Science Teacher Education, 10, 43-54. doi: 10.1023/A:1009424713416

Beardsley, D. C., \& O’Dowd. D. D. (1961). The college-student image of the scientist. Science, 133, 997-1001. doi: 10.1126/science.133.3457.997

Buldu, M. (2006). Young children's perceptions of scientists: A preliminary study. Educational Re-search, 48(1), 121-132. doi: 10.1080/00131880500498602

Calabrese Barton, A. (2001). Science education in urban settings: seeking new ways of praxis through critical ethnography. Journal of Research in Science Teaching, 38, 899-917. doi: 10.1002/tea.1038

Chambers, D. W. (1983). Stereotypic images of the scientist: The Draw-a-Scientist Test. Science Education, 6, 255-265. doi: 10.1002/sce.3730670213

Chavous, T. (2002). African American college students in predominantly White institutions of higher education: Considerations of race and gender. Perspectives, 8, 142-150.

Chavous, T., Rivas-Drake, D., Smalls, C., Griffin, T. \& Cogburn, C. (2008). Gender matters: The differential influences of school racial discrimination and racial identity on academic engagement among African American boys and girls. Developmental Psychology, 44, 637-654.

Chen, J., \& Cowie, B. (2014). Scientists talking to students through videos. International Journal of Science and Mathematics Education, 12(2), 445 - 465. DOI:10.1007/s10763-013-9415-y

Chernobilsky, L.B. (2006), El uso de la computadora como auxiliar en el análisis de datos cualitativos, in Vasilachis de Gialdino, I. (coord.), Estrategias de investigación cualitativa (pp. 239-262), Barcelona: Gedisa.

Christidou, V. (2006). Greek students' science-related interests and experiences: Gender differences and correlations. International Journal of Science Education, 28, 1181-1199. doi: 10.1080/09500690500439389 
Christidou, V., Hatzinikita, V. \& Samaras, G. (2012). The image of scientific researchers and their activity in Greek adolescents' drawings. Public Understanding of Science, 21(5), 626-647. DOI: 10.1177/0963662510383101

Christidou, V. (2011). Interest, attitudes and images related to science: Combining students' voices with the voices of school Science, teachers, and popular science. International Journal of Environmental \& Science Education, 6(2), 141-159.

Comisión Mujeres y Ciencia (2020). Informe mujeres investigadoras. Comisión de Mujeres y Ciencias ,CSIC. Recuperado de

http://mujeresconciencia.com/app/uploads/2020/o7/informe_mujeres_investigadoras-2020.pdf

Comisión Nacional de Ciencia y Tecnología. (2017). Política institucional equidad de género en ciencia y tecnología período 2017-2025. Recuperado de https://www.conicyt.cl/ wp-content/uploads/2015/o3/Politica-Institucional-Equidad-de-Genero-en-CyT-Periodo-2017_2025.pdf

Erten, S., Kıray, S. A., \& Şen-Gümüş, B. (2013). Influence of scientific stories on students ideas about science and scientists. International Journal of Education in Mathematics, Science and Technology, 1(2), 122-137.

Farland-Smith D. (2017) The Evolution of the Analysis of the Draw-a-Scientist Test. In: Katz P. (eds) Drawing for Science Education (pp. 171-178), Rotterdam: SensePublishers.

Finson, K. D. (2001). Applicability of the DAST-C to the images of scientists drawn by students of different racial groups. Journal of Elementary Science Education, 15, 15-26. doi: 10.1007/BFo3174741

Finson, K.D. (2002). Drawing a scientist: What we do and do not know after fifty years of drawings. School Science and Mathematics, 102, 335-345. doi: 10.1111/j.1949-8594.2002. tb18217.x

Finson, K. D., Beaver, J. B. \& Cramond, B. L. (1995). Development and field test of a checklist for the Draw-a-Scientist-Test. School Science \& Mathematics, 95(4), 195-205. doi: 10.1111/j.1949-8594.1995.tb15762.x

Finson, K., Pedersen, J. \& Thomas, J. (2006). Comparing science teaching to students' perception of scientists. School Science and Mathematics, 106(1), 8-15.

Flick, L. (1990). Scientist in residence program improving children's image of science and scientists. School Science and Mathematics, 90, 204-214. doi:10.1111/j.1949-8594.1990. tb15536.x

Fort, D. C. \& Varney, H. L. (1989). How students see scientists: Mostly male, mostly white, and mostly benevolent. Science and Children, 26, 8-13.

Fundación Chile- España. Memorias. Madrid (2015)

Fung, Y.Y.H. (2002). A comparative study of Primary and Secondary School Students' Images of Scientists. Research in Science and Technological Education, 20, 199-213. doi: 10.1080/0263514022000030453

Gonzalez Weil, C., Castillo Fierro, P., Lopez Leiva, V., y Bravo González, P. (2009). Científicos jóvenes y sonrientes: la imagen de científico de los estudiantes chilenos de 11 y 12 grado en diferentes contextos escolares. Enseñanza de Las Ciencias, (Extra), 2541-2545.

Hernández, R., Fernández, C., y Baptista, P. (2010). Metodología de la investigación. CDMX: McGraw-Hill.

Joyce, B. A. \& Farenga, S. J. (1999). Informal science experience, attitudes, future interest in science, and gender of high-ability students: an exploratory study. School Science and Mathematics, 99, 431-437. doi: 10.1111/j.1949-8594.1999.tb17505.x 
Kitzinger, J., Haran, J., Chimba, M. and Boyce, T. (2008a) Role models in the media: an exploration of the views and experiences of women in science, engineering and technology. Cardiff University. Report of the UK Resource Centre for Women in Science, Engineering and Technology (UKRC).

Kitzinger, J., Chimba, M., Williams, A., Haran, J., and Boyce, T. (2008b) Gender, stereotypes and expertise in the press: how newspapers represent male and female scientists . Cardiff University. Report of the UK Resource Centre for Women in Science, Engineering and Technology (UKRC)

Mason, C. L., Kahle, J. B. \& Gardner, A. L. (1991). Draw-A-Scientist Test: Future implications. School Science and Mathematics, 91, 193-198. doi: 10.1111/j.1949-8594.1991.tb12078.x

Manzoli, F., Castelfranchi, Y., Gouthier, D. \& Cannata, I. (2006). Children's perceptions of science and scientists: A case study based on drawings and story-telling. Paper presentado en la gth International Conference on Public Communication of Science and Technology. Seúl.

Mattern, N. \& Schau, C. (2002). Gender differences in science attitude-achievement relationships over time among white middle-school students. Journal of Research in Science Teaching, 39(4), 324-340. doi: 10.1002/tea.10024

Mead, M., \& Metraux, R. (1957). The image of the scientist among high school students: A pilotstudy. Science, 126, 384-390. doi: 10.1126/science.126.3270.384

Medina, W., Middleton, K. \& Orihuela, W. (2011). Using the DAST-C to explore colombian and bolivian students' images of scientists. International Journal of Science and Mathematics Education. 9. 657-690.

Meyer, C., Guenther, L. \& Joubert, M. (2019) The Draw-aScientist Test in an African context: comparing students' (stereotypical) images of scientists across university faculties, Research in Science \& Technological Education, 37(1), 1-14, doi:10.1080/02635143.2018 .1447455

Embajada de Chile en España. (2020). Relaciones bilaterales. Recuperado de https://chile. gob.cl/espana/relacion-bilateral/cooperacion-internacional

Miller, D. I., Eagly, A. H., \& Linn, M. C. (2015). Women's representation in science predicts national gender-science stereotypes: Evidence from 66 nations. Journal of Educational Psychology, 107(3), 631-644.

Morgan, C., Isaac, J. D. \& Sansone, C. (2001). The role of interest in understanding the career choices of female and male college students. Sex Roles, 44, 295-320. doi: 10.1023/A:1010929600004

Narayan, R., Park, S., Peker, D., \& Suh, J. (2013). Students' images of scientists and doing science: An international comparison study. Eurasia Journal of Mathematics, Science \& Technology Education, 9(2), 115-129. doi: 10.12973/eurasia.2013.923a

Neathery, M. F. (1997). Elementary and Secondary Students' Perceptions Toward Science: Correlations with Gender, Ethnicity, Ability, Grade, and Science Achievement. Electronic Journal of Science Education, 2, 1. Recuperado de http://ejse.southwestern.edu/article/ view/7573/5340 (30/04/2016)

Newton, P. D. \& Newton, L. D. (1992). Young children's perceptions of science and the scientist. International Journal of Science Education, 14.(3), 331-348. doi: 10.1080/0950069920140309

Reinisch, B., Krell, M., Hergert, S., Gogolin, S. \& Krüger, D. (2017), Methodical challenges concerning the Draw-A-Scientist Test: a critical view about the assessment and evaluation of learners' conceptions of scientists, International Journal of Science Education, 39(14), 1952-1975. 
Rodari, P. (2007). Science and scientists in the drawings of European children. Journal of Science Communication, 6(3), 1-12.

Rubbia, G., D’Addezio, G., Marsili, A. y A. Carosi (2015). Science and scientists from a child's point of view: an overview from drawings. Geological Society, London, Special Publications, 419, 161-170,

Rubin, E., Bar, V., Cohen, A. (2010). The images of scientists and science among Hebrewand Arabic-speaking pre-service teachers in Israel. International Journal of Science Education, 25(7), 821-846.

Ruiz-Mallén, I. \& Escalas, M. T. (2012). Scientists Seen by Children: A Case Study in Catalonia, Spain. Science Communication, 34(4), 520-545. doi: 10.1177/1075547011429199

Schibeci, R. A. \& Riley, J. P. (1986). Influence of students' background and perceptions on science attitudes and achievement. Journal of Research in Science Teaching, 23(3), 177-187. doi: 10.1002/tea.3660230302

Schibeci, R. A. \& Sorenson, I. (1983). Elementary school children's perceptions of scientists. School Science and Mathematics, 83(1), 14-19. doi: 10.1111/j.1949-8594.1983.tb10087.x

She, H. C. (1998). Gender and grade level differences in Taiwan students' stereotypes of science and scientists. Research in Science and Technological Education, 16(2), 125-135. doi: $10.1080 / 0263514980160203$

Steinke, J.; M. Long; M. J. Johnson \& S. Ghosh (2008). Gender Stereotypes of Scien- tist Characters in Television Programs Popular Among Middle School-Aged Children, presentado en la Annual Meeting of the Association for Education in Journalism and Mass Communication, Chicago.

Song, J., \& Kim, K.S. (1999). How Korean students see scientists: the images of the scientist. International Journal of Science Education, 21(9), 957-977. doi: 10.1080/o95006999290255

Sumrall, W. J. (1995). Reasons for the perceived images of scientists by race and gender of students in grades 1-7. School Science and Mathematics, 9(2), 83-90. doi: 10.1111/j.19498594.1995.tb15733.x

Toğrol, A. Y. (2013). Turkish students' images of scientist. Journal of Baltic Science Education, 12(3), 289-298.

Toma, R. B., Greca, I. M.\& Orozco Gómez, M. L. (2018) Una revisión del protocolo Draw-aScientist-Test (DAST). Revista Eureka sobre Enseñanza y Divulgación de las Ciencias,15(3), 3104. doi:10.25267/Rev_Eureka_ensen_divulg_cienc.2018.v15.i3.3104

Oaklander V. (2008). El tesoro escondido. La vida interior de niños y adolescentes. SantiagoChile: Editorial Cuatro Vientos.

Valderrama, L.B., Vernal-Vilicic, T.P \& Méndez-Caro, L. (2016). Represen- tación infantil de la ciencia usando el test Dibujando un Científico (DAST). Posibilidades de cambio desde la comunicación científica. Infor- mación Tecnológica, 27(6), 203-214. doi: 10.4067/ So718-0764,2016000600021

Vernal, T. (2015). La comunicación científica para el desarrollo cultural y económico: el caso de las potencialidades astronómicas de la Región de Antofagasta en Chile. Cuadernos. info, (37), 213-224. doi: 10.7764/cdi.37.691

Vernal, T. \& Valderrama, L.B. (2014). La percepción de la ciencia y de cien- tíficos/as en un país austral. El caso de DeLTA Chile. Tercer Milenio, (28), 4,2-50.

Weinburgh, M. H. (2003). The effects of systemic reform on urban, African American fifth grade students' attitudes toward science. Journal of Women and Minorities in Science and Engineering, 9(1), 53-72. doi: 10.1615/JWomenMinorScienEng.v9.i1.40

Weinburgh, M. H. \& Steele, D. (2000). The modified attitudes toward science inventory: 
Developing an instrument to be used with fifth grade urban students. Journal of Women and Minorities in Science and Engineering, 6(1), 87-98.

Weingart P, Pansegrau P (2003). Perception and Representation of Science in Literature and Fiction-Film: Introduction. Public Understanding of Science 12(3): 227-228.

Woods-Townsend, K., Christodoulou, A., Rietdijk, W., Byrne, J., Griffiths, J. \& Grace, M. (2015). Meet the Scientist: The Value of Short Interactions Between Scientists and Students. International Journal of Science Education, 6(1), 89-113. doi: 10.1080/21548455.2015.1016134

Zhai, J., Jocz, J. A., Tan \& Aik-Ling. (2014). Am I Like a Scientist?: Primary children's images of doing science in school. International Journal of Science Education, 36(4), 553-576. doi:10.1080/09500693.2013.791958 
